BNL-45268

AD/RHIC-82

Informal Report

BNL --45268

DE9 1001132

\title{
The Residual Tune Splitting in the Presence of Linear Coupling
}

\author{
G. Parzen
}

\section{October 1990}

\author{
DISCLAIMER
}

\begin{abstract}
This report was prepared as an account of work sponsored by an agency of the United States Government. Neither the United States Government nor any agency thereof, nor any of their employees, makes any warranty, express or implicd, or assumes any legal liability or responsibility for the accuracy, completeness, or usefulness of any information, apparatus, product, or process disclosed, or represents that its use would not infringe privately owned rights. Reference herein to any specific commercial product, process, or service by trade name, trademark, manufacturer, or otherwise does not necessarily constitute or imply its endorsement, recommendation, or favoring by the United States Government or any agency thereof. The views and opinions of authors expressed herein do not necessarily state or reflect those of the United States Government or any agency thereof.
\end{abstract}

\section{R H I C P R O J E C T}

\author{
Brookhaven National Laboratory \\ Associated Universities, Inc. \\ Upton, NY 11973
}

\section{Under Contract No. DE-AC02-76CH 00016 with the UNITED STATES DEPARTMENT OF ENERGY}

DISTPIBUTIONN OF THIS DOCUMENT IS UNLIMITE 


\title{
The Residual Tune Splitting in the Presence of Linear Coupling
}

\author{
G. Parzen
}

\section{Introduction}

The presence of random skew quadirupole field errors will couple the $x$ and $y$ motions. The $x$ and $y$ motions are then each given by the sum of 2 normal modes with the tunes $\nu_{1}$ and $\nu_{2}$, which may differ appreciably from $\nu_{x}$ and $\nu_{y}$, the unperturbed tunes. This is often called tune splitting since $\left|\nu_{1}-\nu_{2}\right|$ is usually larger than $\left|\nu_{x}-\nu_{y}\right|$. This tune splitting may be large in proton accelerators using superconducting magnets, because of the relatively large random skew quadrupole field errors that are expected ir these magnets. This effects is also increased by the required insertions in proton colliders which generate large $\beta$-functions in the insertion region.

This tune splitting has been studied in the RHIC accelerator (the Relativistic Heavy Ion Collider proposed at Brookhaven National Laboratory). For RHIC, a tune splitting as large as 0.2 was found in one worse case. A correction system has been developed ${ }^{1}$ for correcting this large tune splitting which uses two families of skew quadrupole correctors. It has been found ${ }^{1}$ that this correction system corrects most of the large tune splitting, but a residual tune splitting remains that is still appreciable.

RHIC has to operate within a box in tune space whose width is $\Delta \nu=33 \times 10^{-3}$ in order to avoid all resonances which are tenth order or less. It appears desirable to correct the tune splitting to a level which is much smaller than $33 \times 10^{-3}$. The residual tune splitting that remained after correction with the 2 family tune splitting correction system was found to be about $18 \times 10^{-3}$.

The residual tune splitting appears to be due to higher order effects of t'ie random $a_{1}$ multipole. Loosely speaking, one may say that the residual tune splitting is associated with the nearby linear sum resonance, $\nu_{x}+\nu_{y}=$ integer. A skew quadrupole correction system has been developed that appears able to correct a large part of the residual tune splitting. This consists of skew quadrupole correctors near the high- $\beta$ quadrupoles ir the insertions, which are excited so as to generate the harmonics that would drive the nearby sum resonances, $\nu_{x}+\nu_{y}=$ integer.

1 G. Parzen, BNL Report AD/RHIC-AP-72 (1988). 


\section{Calculation and Correction of the Normal Mode Tune}

The presence of the random skew quadrupole errors given by the $a_{1}$ multipoles in each magnet will couple the $x$ and $y$ motions. The $x$ and $y$ motion are then each given by the sum of 2 normal modes which have the tunes $\nu_{1}$ and $\nu_{2}$.

The normal mode tunes $\nu_{1}$ and $\nu_{2}$ can be computed in the following way. The effect of the $a_{1}$ multipole in each magnet is represented by putting point $a_{1}$ multipoles at the entrance and exit of each magnet, with half the strength of the $a_{1}$ multipole. The one turn transfer matrix around the ring can be computed by multiplying the transfer matrices of all the magnets and all the point $a_{1}$ multipoles in the ring. The $4 \times 4$ one turn transfer matrix can be written as

$$
T=\left[\begin{array}{ll}
M & n \\
m & N
\end{array}\right]
$$

where $M, N, m, n$ are each $2 \times 2$ matrices. The tunes $\nu_{1}$ and $\nu_{2}$ can then be computed from the following equations ${ }^{2}$

$$
\begin{aligned}
\cos \mu_{1}+\cos \mu_{2} & =\frac{1}{2} \operatorname{Tr}(M+N) \\
\cos \mu_{1}-\cos \mu_{2} & =\left\{\left[\frac{1}{2} \operatorname{Tr}(M-N)\right]^{2}+|m+\bar{n}|\right\}^{\frac{1}{2}} \\
\bar{n} & =\left[\begin{array}{cc}
n_{22} & -n_{12} \\
-n_{21} & n_{11}
\end{array}\right], \\
\mu_{1} & =2 \pi \nu_{1}, \quad \mu_{2}=2 \pi \nu_{2} .
\end{aligned}
$$

$\operatorname{Tr}(M)$ is the trace of $M .|M|$ is the determinant of $M$.

Treating the random $a_{1}$ as a perturbation, one can find an approximate result which is valid close to the resonance line $\nu_{x}-\nu_{y}=0$ where $\nu_{x}, \nu_{y}$ are the tunes in the absence of the $a_{1}$ fields,

$$
\begin{aligned}
\nu_{1}-\nu_{2} & =2\left\{\left[\frac{1}{2}\left(\nu_{x}-\nu_{y}\right)\right]^{2}+|\Delta \nu|^{2}\right\} \\
\nu_{1}+\nu_{2} & =\nu_{x}+\nu_{y} \\
\Delta \nu & =\frac{1}{4 \pi \rho} \int d s\left(\beta_{x} \beta_{y}\right)^{\frac{1}{2}} a_{1} \exp \left(i \bar{\nu}\left(\psi_{x} / \nu_{x}-\psi_{y} / \nu_{y}\right)\right) \\
\bar{\nu} & =\frac{1}{2}\left(\nu_{x}+\nu_{y}\right)
\end{aligned}
$$

$\Delta \nu$ may be regarded as the width of the nearby coupling resonance.

${ }^{2}$ E.D. Courant and H.S. Snyder, Ann. Phys. 3, 1 (1958). 
Equations (2.3) show that when $\nu_{x} \simeq v_{y}$, the shift in the $\nu$-values looks like a tune splitting. Equations (2.3) also suggest that if the $a_{1}$ correctors are set so as to make $\Delta \nu=0$, then the tune splitting is minimized. This is not a practical way to set the $a_{1}$ correctors as the $\Delta \nu$ and the $a_{1}$ errors are not known for a real accelerator. Usually the $a_{1}$ correctors are set so as to minimize the tune splitting which can be measured. The results from this way of setting the $a_{1}$ correctors is discussed in Section 3. It is instructive to see what happens when the $a_{1}$ correctors are set to inake $\Delta \nu=0$. These results are shown in Table 1 for 10 distributions of $a_{1}$ errors and for two RHIC lattices, one having $\beta^{*}=2$ at all 6 crossing points and one having $\beta^{*}=6$ at all 6 crossing points.

The results in Table 1 were found using the RHIC lattices with the expected random quadrupole errors, $a_{1}$ and $b_{1}$. These quadrupoles errors are listed in Section 4 . The tune of the normal modes, $\nu_{1}$ and $\nu_{2}$, are computed using the exact result, Eq. (2.2). The tune shift due to the random $b_{1}$ has been corrected using the $\mathrm{QF}$ and $\mathrm{QD}$ quadrupoles in the regular cells. A correction system has been developed ${ }^{1}$ for correcting the tune splitting which uses two families of skew quadrupoles as correctors. Results are given for $\nu_{1}, \nu_{2}$ before and after the application of the tune splitting correction, and are listed as the uncorrected and corrected cases. The tune splitting correction was set so as to make $\Delta \nu=0$, where $\Delta \nu$ is the $\nu_{x}=\nu_{y}$ coupling resonance width given in Eqs. (2.3).

The unperturbed $\nu_{x}, \nu_{y}$ for RHIC for Table 1 is $\nu_{x}=28.826, \nu_{y}=28.821$. Only the fractional part of the $\nu$-values are listed in Table 1.

The largest tune splittings found in Table 1 are

$\begin{array}{lcl}\left|\nu_{1}-\nu_{2}\right| / 10^{-3}, \beta^{*}=6 & \underline{\text { Uncorrected }} & \\ \left|\nu_{1}-\nu_{2}\right| / 10^{-3}, \beta^{*}=2 & 91 & \text { Corrected } \\ & 228 & 23\end{array}$

One sees that the tune splitting remaining after correction is considerably larger than the $5 \times 10^{-3}$ predicted by Eq. (2.3). The remaining tune splitting after correction is called the residual tune splitting in this paper. It appears to be due to higher order terms in $a_{1}$ which are omitted in Eq. (2.3).

The presence of higher order effects of the random $a_{1}$ can be seen by plotting $\nu_{1}$ and $\nu_{2}$ against $a_{1}$ and comparing this result with that predicted by the approximate equations Eqs. (2.3). Figure 1 shows this plot for the uncorrected case for the field error distribution number 2 and $\beta^{*}=2$. To simplify the comparison with Eqs. (2.3) the unperturbed $\nu$ values, $\nu_{x}$ and $\nu_{y}$ were moved on to the $\nu_{x}=\nu_{y}$ resonance to $\nu_{x}=0.824, \nu_{y}=0.824$. Also, the random $b_{1}$ error was omitted in this study. In Fig. $1, a_{1}-0.8$ means that the random $a_{1}$ in every magnet was reduced by the factor 0.8 . 
Table 1: Results for the correction of the tune splitting for two RHIC lattices using a 2 family tune splitting correction system set to make $\Delta \nu=$ 0 , where $\Delta \nu$ is the width of the difference resonance $\nu_{x}=\nu_{y}$.

\begin{tabular}{|c|c|c|c|c|c|c|c|}
\hline & \multicolumn{3}{|c|}{-Uncorrected- } & & \multicolumn{3}{|c|}{ - Corrected - } \\
\hline $\begin{array}{l}\text { Error } \\
\text { Field } \\
\text { Dist. }\end{array}$ & $\nu_{1}$ & $\nu_{2}$ & $\left|\nu_{1}-\nu_{2}\right| / 10^{-3}$ & & $\nu_{1}$ & $\nu_{2}$ & $\left|\nu_{1}-\nu_{2}\right| / 10^{-3}$ \\
\hline & \multicolumn{7}{|c|}{$\beta^{*}=6$} \\
\hline 1 & 0.842 & 0.806 & 36 & 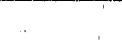 & 0.825 & 0.819 & 6 \\
\hline 2 & 0.784 & 0.863 & 21 & & 0.822 & 0.812 & 10 \\
\hline 3 & 0.851 & 0.796 & 55 & & 0.826 & 0.820 & 6 \\
\hline 4 & 0.785 & 0.865 & 80 & & 0.825 & 0.815 & 9 \\
\hline 5 & 0.813 & 0.836 & 23 & & 0.831 & 0.818 & 13 \\
\hline 6 & 0.830 & 0.819 & 11 & & 0.828 & 0.820 & 9 \\
\hline 7 & 0.830 & 0.822 & 8 & & 0.829 & 0.822 & 7 \\
\hline 8 & 0.778 & 0.869 & 91. & & 0.820 & 0.805 & 15 \\
\hline 9 & 0.809 & 0.839 & 30 & & 0.827 & 0.821 & 6 \\
\hline \multirow[t]{2}{*}{10} & 0.845 & 0.803 & 42 & & 0.827 & 0.821 & 6 \\
\hline & \multicolumn{7}{|c|}{$\beta^{*}=2$} \\
\hline 1 & 0.796 & 0.854 & 59 & & 0.828 & 0.823 & 6 \\
\hline 2 & 0.707 & 0.935 & 228 & & 0.838 & 0.819 & 19 \\
\hline 3 & 0.869 & 0.783 & 86 & & 0.825 & 0.829 & 4 \\
\hline 4 & 0.772 & 0.883 & 111 & & 0.831 & 0.823 & 7 \\
\hline 5 & 0.779 & 0.872 & 93 & & 0.836 & 0.820 & 16 \\
\hline 6 & 0.848 & 0.805 & 43 & & 0.832 & 0.821 & 11 \\
\hline 7 & 0.840 & 0.847 & 7 & & 0.852 & 0.834 & 18 \\
\hline 8 & 0.742 & 0.895 & 153 & & 0.838 & 0.818 & 20 \\
\hline 9 & 0.785 & 0.866 & 81 & & 0.828 & 0.823 & 6 \\
\hline 10 & 0.749 & 0.891 & 142 & & 0.823 & 0.827 & 5 \\
\hline
\end{tabular}

The results in Fig. 1 are in good agreement with Eqs. (2.3). Since $\nu_{x}=\nu_{y}$, Eqs. (2.3) predict that $\nu_{1}$ and $\nu_{2}$ should be linear in $a_{1}$, and $\nu_{a v}=\left(\nu_{1}+\nu_{2}\right) / 2$ should be constant.

Figure 2 shows a similar plot for the corrected case. The 2 family tune splitting correction system has been set to make $\Delta \nu=0$, where $\Delta \nu$ is the $\nu_{x}=\nu_{y}$ coupling resonance width given in Eq. (2.3). The results in Fig. 2 are not in agreement with Eqs. (2.3) showing the presence of higher order effects due to $a_{1}$. Both $\nu_{1}, \nu_{2}$ and $\nu_{a v}=\left(\nu_{1}+\nu_{2}\right) / 2$ appear to have an $a_{1}^{3 / 2}$ dependence on $a_{1} . \nu_{a v}$ is not constant, and the $\nu_{1}$ and $\nu_{2}$ shifts are not equal as predicted by Eq. (2.3). 


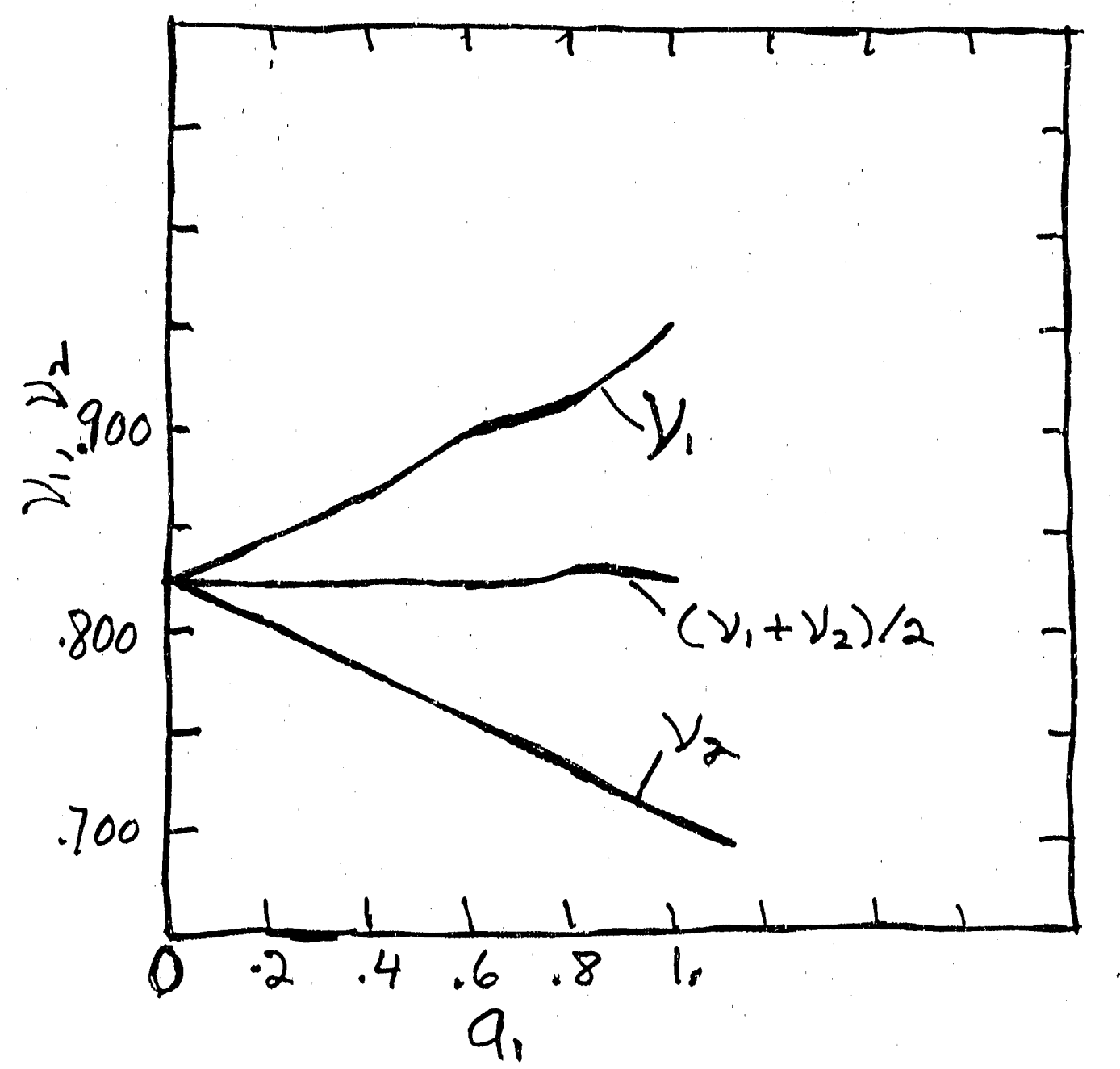

Figure 1: A plot of the normal mode tunes, $\nu_{1}$ and $\nu_{2}$, versus the size of the random skew quadrupole error, $a_{1}$ for a particular distribution of the random $a_{1}$. The tune splitting was not corrected. 


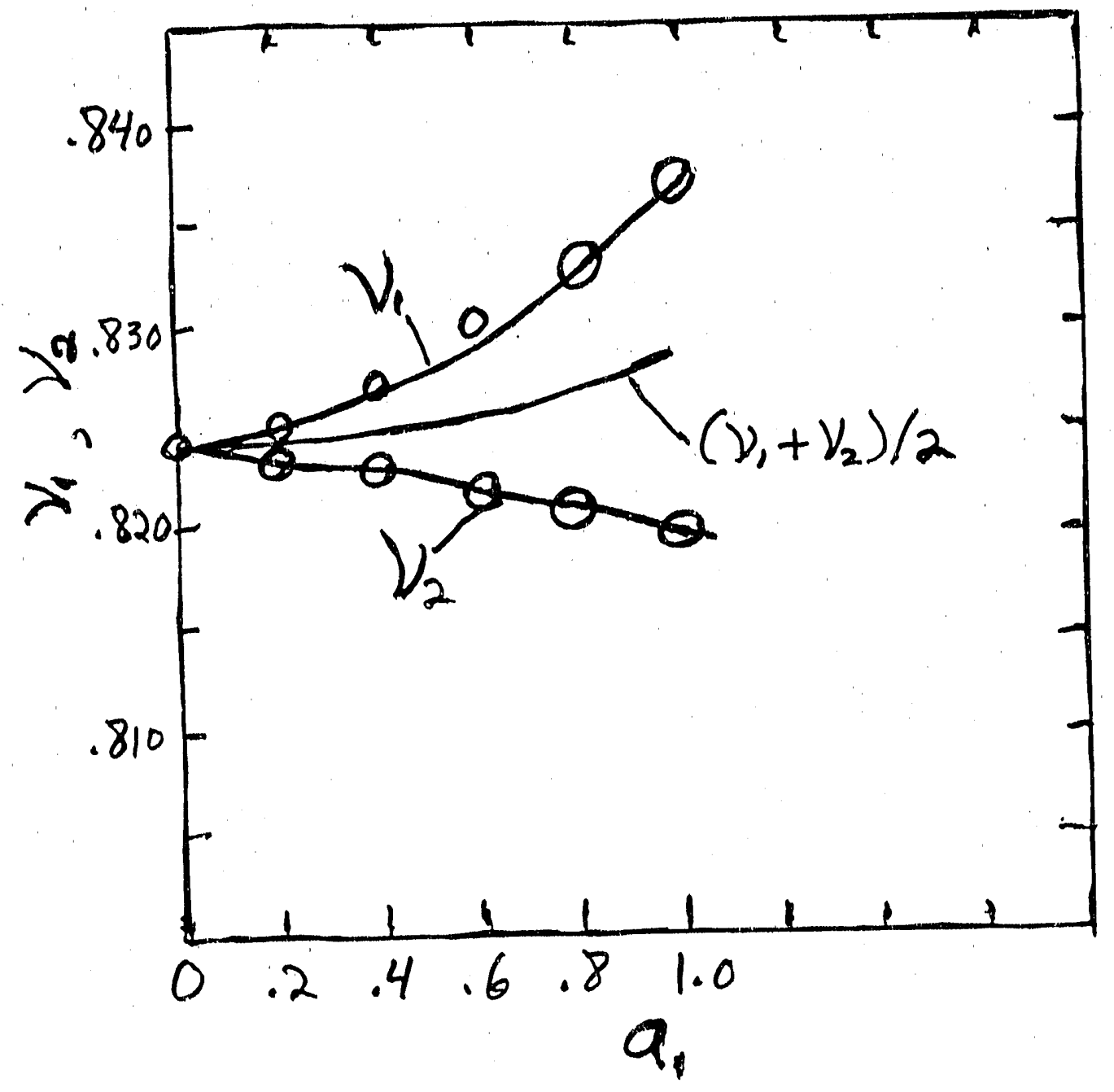

Figure 2: A plot of the normal mode tunes, $\nu_{1}$ and $\nu_{2}$ versus the size of the random skew quadrupole error, $a_{1}$. The tune splitting has been corrected with the 2 family correction system. The open circles show the points on an $a_{1}^{3 / 2}$ curve. 
Using the computed results and Eqs. (2.3), one may conjecture that the higher order effects may be described by the following equation;

$$
\begin{aligned}
& \left(\nu_{1}-\nu_{2}\right) / 2=\left\{\left[\left(\nu_{x}-\nu_{y}\right) / 2\right]^{2}+|\Delta \nu|^{2}+g_{-}\right\}^{1 / 2}, \\
& \left(\nu_{1}+\nu_{2}\right) / 2=\left\{\left[\left(\nu_{x}+\nu_{y}\right) / 2\right]^{2}+g_{+}\right\}^{1 / 2}
\end{aligned}
$$

where $g_{-}$and $g_{+}$are two functions of $a_{1}$ which vary like $a_{1}^{3}$.

\section{Correction of the Residual $\left|\nu_{1}-\nu_{2}\right|$}

A first step in reducing the residual $\left|\nu_{1}-\nu_{2}\right|$, is to set the $a_{1}$ correctors of the two family tune splitting correction system to minimize the residual $\left|\nu_{1}-\nu_{2}\right|$, instead of setting them to make $\Delta \nu=0$ as indicated by Eqs. (2.3). The results of doing this are given in Table 2. In Table 2, the results for the residual $\left|\nu_{1}-\nu_{2}\right|$ listed in Table 1 using the $\Delta \nu=0$ correction procedure are compared with the results found using the minimizing $\left|\nu_{1}-\nu_{2}\right|$ procedure for the same 10 distributions of random $a_{1}$ and $b_{1}$ errors and for the $\beta^{*}=6$ and $\beta^{*}=2$ lattices for RHIC.

Table 2 shows that the minimizing $\left|\nu_{1}-\nu_{2}\right|$ procedure considerably reduces the residual $\left|\nu_{1}-\nu_{2}\right|$ for some field error distributions. A possible explanation is that for some error distributions, the setting of the correctors to make $\Delta \nu=0$ excites the harmonics in $a_{1}$ that drive the nearby sum resonances $\nu_{x}+\nu_{y}=$ integer, and it is these harmonics that are believed to be responsible for the residual $\left|\nu_{1}-\nu_{2}\right|$.

One may note that after correction, not only is there a considerable residual $\left|\nu_{1}-\nu_{2}\right|$ for snme field error distributions, but also the average tune $\nu_{a v}=\left(\nu_{1}+\nu_{2}\right) / 2$ may be considerably off from $\left(\nu_{x}+\nu_{y}\right) / 2$. It was found that the location of $\nu_{a v}$ may be corrected using the normal tune correction system which in RHIC consists of the QF and QD quadrupoles in the regular cells of the arcs which are corrected in two families. This is indicated by Eqs. (2.3). However the normal tune correction system will not change the residual $\left|\nu_{1}-\nu_{2}\right|$ by much.

To further reduce the residual tune splitting requires additional $a_{1}$ correctors so that one can control not only the harmonics in $a_{1}^{n}$ that drive the difference resonance, $\nu_{x}-\nu_{y}$, but also the harmonics in $a_{1}$ that would drive the nearby sum resonances, $\nu_{x}+\nu_{y}=$ integer, which for RHIC are principally the 58 and 57 harmonics in $a_{1}$. The most effective place to put these correctors is near the quadrupoles in the insertions that have high $\beta$-functions. In RHIC which has 6 beam crossing points, there are 12 such high $\beta$ locations. The two family tune splitting correction system ${ }^{1}$ has $a_{1}$ correctors at 6 of the high $\beta$ locations. The 
Table 2: Residual tune splitting resulting from two different methods of tune splitting correction using the 2 -family tune splitting correction system.

\begin{tabular}{|c|c|c|}
\hline $\begin{array}{c}\text { Error } \\
\text { Field } \\
\text { Distribution } \\
\end{array}$ & $\begin{array}{c}\left|\nu_{1}-\nu_{2}\right| / 10^{-3} \\
\Delta \nu=0 \\
\text { Correction }\end{array}$ & $\begin{array}{c}\left|\nu_{1}-\nu_{2}\right| / 10^{-3} \\
\text { minimize }\left|\nu_{1}-\nu_{2}\right| \\
\text { Correction }\end{array}$ \\
\hline & \multicolumn{2}{|c|}{$\beta^{*}=6$} \\
\hline 1 & 6 & 6 \\
\hline 2 & 10 & 6 \\
\hline 3 & 6 & 6 \\
\hline 4 & 9 & 6 \\
\hline 5 & 13 & 9 \\
\hline 6 & 9 & 7 \\
\hline 7 & 7 & 6 \\
\hline 8 & 15 & 7 \\
\hline 9 & 6 & 5 \\
\hline \multirow[t]{2}{*}{10} & 6 & 6 \\
\hline & \multicolumn{2}{|c|}{$\beta^{*}=2$} \\
\hline 1 & 6 & 5 \\
\hline 2 & 19 & 18 \\
\hline 3 & 4 & 3 \\
\hline 4 & 7 & 7 \\
\hline 5 & 16 & 8 \\
\hline 6 & 11 & 11 \\
\hline 7 & 18 & 7 \\
\hline 8 & 20 & 16 \\
\hline 9 & 6 & 6 \\
\hline 10 & 5 & 4 \\
\hline
\end{tabular}

proposed enlargement of the $a_{1}$ correctors is to have $a_{1}$ correctors at all 1 ? of the high $\beta$ locations, and all $12 a_{1}$ correctors are separately powered.

A computer study was done to evaluate the performance of the enlarged $a_{1}$ correction system in reducing the residual $\left|\nu_{1}-\nu_{2}\right|$. The results are given in Table 3 for one particular method of using the $a_{1}$ correctors which is described below. One may note that the unperturbed tune of the accelerator in this study has a tune splitting of $\left|\nu_{x}-\nu_{y}\right|=5 \times 10^{-3}$, and the results listed in Table 3 for tho residual $\left|\nu_{1}-\nu_{2}\right|$ using the enlarged $a_{1}$ correction are good.

The method used to reduce the residual $\left|\nu_{1}-\nu_{2}\right|$ is the following: the $6 a_{1}$ correctors that make up the 2-family tune splitting correction system are corrected in the usual way as described in Ref. 1. This provides two variables which are primarily used to correct the 
Table 3: The reduction in the residual tune splitting obtained using the enlarged $a_{1}$ correction systern, compared with the restilts obtained with the 2-family correction system, minimizing $\left|\nu_{1}-\nu_{2}\right|$.

\begin{tabular}{|c|c|c|}
\hline $\begin{array}{c}\text { Error } \\
\text { Fidd } \\
\text { Distribution }\end{array}$ & $\begin{array}{c}\left|\nu_{1}-\nu_{2}\right| / 10^{-3} \\
2 \text { Family } \\
\text { Correction } \\
\text { System }\end{array}$ & $\begin{array}{c}\left|\nu_{1}-\nu_{2}\right| / 10^{-3} \\
\text { enlarged } a_{1} \\
\text { Correction } \\
\text { System }\end{array}$ \\
\hline & \multicolumn{2}{|c|}{$\beta^{*}=6$} \\
\hline 1 & 5 & 5 \\
\hline 2 & 8 & 5 \\
\hline 3 & 4 & 4 \\
\hline 4 & 5 & 4 \\
\hline 5 & 12 & 7 \\
\hline 6 & 5 & 5 \\
\hline 7 & 7 & 5 \\
\hline 8 & 6 & 1 \\
\hline 9 & 5 & 4 \\
\hline \multirow[t]{2}{*}{10} & 5 & 5 \\
\hline & \multicolumn{2}{|c|}{$\beta^{*}=2$} \\
\hline 1. & 5 & 5 \\
\hline 2 & 16 & 6 \\
\hline 3 & 3 & 3 \\
\hline 4 & 5 & 5 \\
\hline 5 & 5 & 5 \\
\hline 6 & 9 & 3 \\
\hline 7 & 14 & 4 \\
\hline 8 & 10 & 2 \\
\hline 9 & 6 & 3 \\
\hline 10 & 1 & 3 \\
\hline
\end{tabular}

effects of the difference resonance $\nu_{x}=\nu_{y}$. In addition the $12 a_{1}$ correctors near the high $\beta$ quadrupoles are excited according to the equation

$$
a_{1}=A \cos \left(\psi_{x}+\psi_{y}\right)+B \sin \left(\psi_{x}+\psi_{y}\right)
$$

where $\psi_{x}, \psi_{y}$ are the betatron phases at the correctors and $A$ and $B$ aro two so far unknown variables. This provides 2 more variables used primarily to correct the effects associated with the noarby sum resonances $\nu_{x}+\nu_{y}=$ integer. Using the above hook up of the $a_{1}$ correctors, the 4 available variables are adjusted to minimize $\left|\nu_{1}-\nu_{2}\right|$. This gives the results in Tablo 3 . 
The hook up of the $a_{1}$ correctors described above does not use the full flexibility of the erlarged $a_{1}$ correction system, but it appears to do a fairly good job of reducing the rosidual $\left|\nu_{1}-\nu_{2}\right|$.

There appears to bo some advantages to having some $c_{1}$ correctors in the arcs of the accelerator. The $a_{1}$ correctors in the arcs cannot usually entirely correct the difference resonance as they tend to have about the same value of $\psi_{x}-\psi_{y}$ if they are put near the quadrupoles in the arcs. However if the phase is defined so that $\psi_{x}-\psi_{y} \simeq 0$ at the quadrupoles, then the arc $a_{1}$ correctors can bo used to correct the real part of $\Delta \nu$, the width of the difference resonance $\nu_{x}=\nu_{y}$, which is often a large part of $\Delta \nu$. The strength required of the $a_{1}$ correctors in the insertions is then reduced, and there is some improvement in the performance of the correction system. In RHIC, it is proposed to have $a_{1}$ correctors near most of the defocusing quadrupoles in the arcs which are connected in series in one fumily, which would be used to correct the real part of $\Delta \nu$, the width of the difference resonance. For the results given in Table 3, the arc $a_{1}$ correctors were also used.

The random skew quadrupole errors also produce an appreciable change ${ }^{3}$ in the $\beta$ functions of the normal modes, $\beta_{1} \beta_{2}$. The change in $\beta_{1}, \beta_{2}$ can be quite large for some distributions of random $a_{1}$ errors, as much as $100 \%$ has been found for RHIC. The above described enlarged $a_{1}$ corrector system has been found to be able to also correct most of the change in $\beta_{1}, \beta_{2}$.

\section{Random $a_{1}, b_{1}$ Present in RHIC}

The random quadrupole errors are due to a number of sources that include construction errors in the magnet coils, effective length errors in the quadrupoles, and rotational errors in the positioning of the quadrupoles.

$a_{1}$ and $b_{1}$ are defined so that the field due to $a_{1}$ and $b_{1}$ on the median plane is given by

$$
\begin{aligned}
& B_{y}=B_{0} b_{1} x \\
& B_{x}=B_{0} a_{1} x
\end{aligned}
$$

where $B_{0}$ is the main dipole field.

\footnotetext{
3 G. Parzen, BNL Report AD/RHIC-73 (1990).
} 
The rms random $a_{1}, b_{1}$ used in this study are given in the following table.

\begin{tabular}{lll} 
Source & $\begin{array}{l}a_{1} / 10^{-5} \\
\left(\mathrm{~cm}^{-1}\right)\end{array}$ & \multicolumn{1}{c}{$\begin{array}{l}b_{1} / 10^{-5} \\
\left(\mathrm{~cm}^{-1}\right)\end{array}$} \\
\hline Dipole coil error & 16.8 & 8.4 \\
Quadrupole coil error & 15 & 15 \\
Quadrupole effective length & - & 40 \\
Quadrupole rotation error & 40 & - \\
\hline
\end{tabular}

The assumed effective length error in the quadrupoles is $\Delta L / L=2 \times 10^{-3} \mathrm{rms}$. The assumed rotational error in the quadrupoles is $\Delta \theta=1 \times 10^{-3} \mathrm{rad}$ rms. 

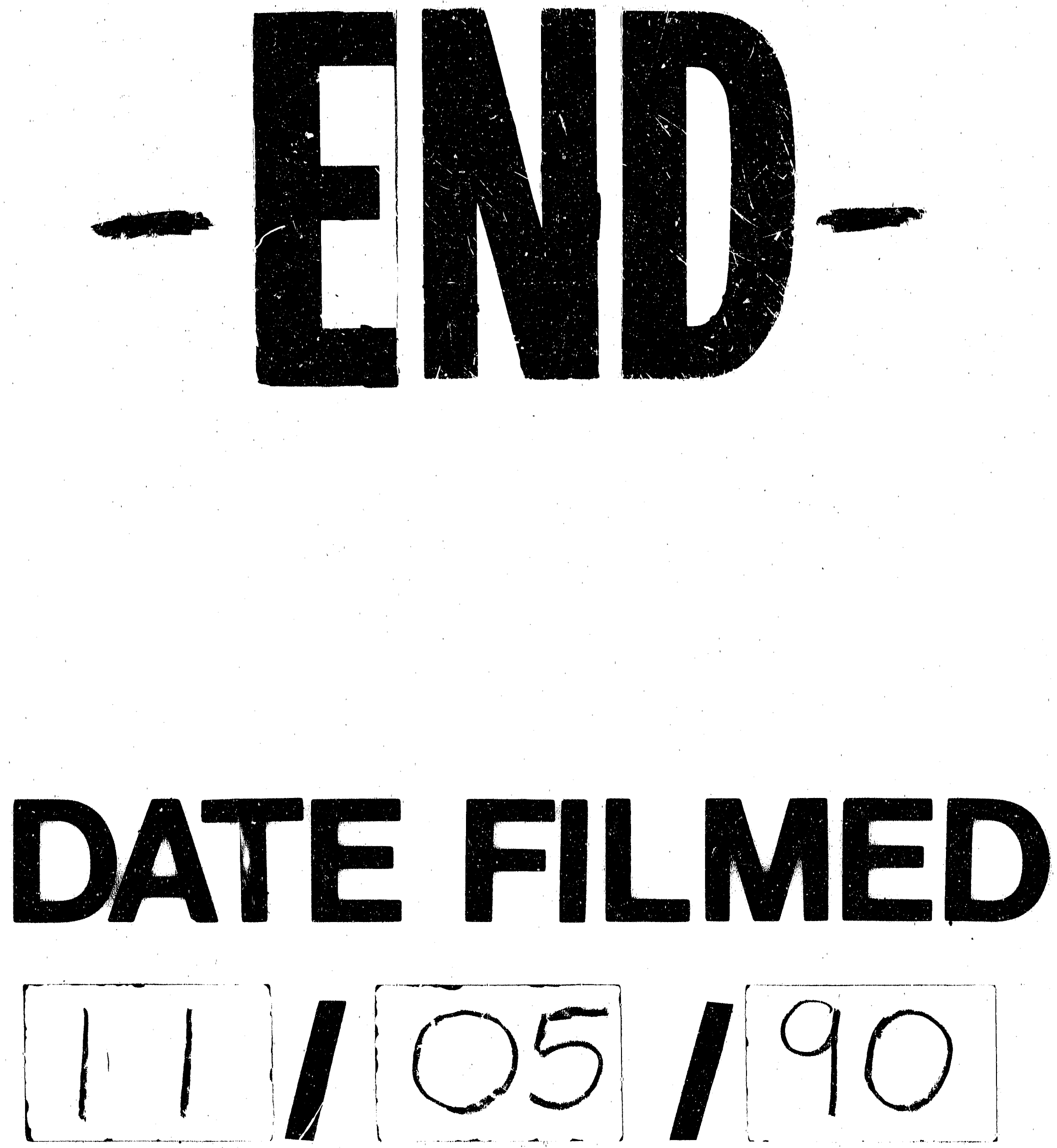
